\title{
Improved EBSD Map Fidelity through Re-indexing of Neighbor Averaged Patterns
}

Stuart I. Wright ${ }^{1}$, Matthew M. Nowell ${ }^{1}$, Scott P. Lindeman ${ }^{1}$ and Patrick Camus ${ }^{2}$

${ }^{1 .}$ EDAX, Draper, Utah, USA

2. EDAX, Mahwah, New Jersey, USA

The Hough transform coupled with triplet indexing [1] has proven very robust in the automated determination of crystallographic orientation from EBSD patterns. However, when the incoming patterns are noisy the ability of these routines to correctly determine the orientation correctly can be compromised. This leads to noisy maps reconstructed from the orientation data, an extreme case is shown in Figure 1(a). A variety of clean-up routines have been developed to find isolated points and assign them orientations generally based on some correlation to the orientations of the surrounding pixels in the scan grid. As the original scan data becomes more and more noisy these clean-up routines [2] tend to produce more and more artifacts. A new approach to re-indexing of the stored patterns has been developed that recovers both non-indexed and mis-indexed points while reducing the number of artifacts. A confirmation of this capability can be observed in Figure 1(b). This approach is based on averaging the pattern at a scan point with the patterns of the point's neighbors.

The details of this re-indexing approach and its limitations will be presented along with results obtained on four different samples:

1) A nickel alloy sample where increasing amounts of artificial noise have been added to the patterns.

2) A nickel alloy sample with varying EBSD camera settings producing patterns with different degrees of noise.

3) A heavily deformed magnesium sample.

4) Fine grained steel wire.

Figure 1 shows the results obtained from the first sample with 20 iterations of applied Poisson noise. Figure 2 shows a summary of the results obtained on samples with varying degrees of camera noise. The results show the positive impact of the pattern averaging on re-indexing although not as dramatic as that achieved on the artificially noisy patterns. The averaging of the pattern at a point with the patterns of neighboring points is similar to increasing the size of the interaction volume. This effect is evident on the results obtained on the heavily deformed magnesium sample. On this sample the averaging led to a loss of fine structure in the resulting map. However, by judicious combining of the original results with the results obtained after averaging enables the advantages of the conventional indexing approach and pattern averaged approach to be realized.

The results obtained on samples (1) and (2) were compared against a new indexing method based on matching of the experimental patterns with a dictionary of simulated patterns [3] as a baseline. This comparison confirmed the accuracy of the results as well as verified the indexing success metric based on the confidence index [4]. 


\section{References:}

[1] SI Wright and BL Adams, Metall Trans A 23 (1992), pp. 759-767.

[2] SI Wright, Mater Sci Technol 22 (2006), pp. 1287-1296.

[3] YH Chen et al, Microsc Microanal (2015), under review.

[4] DP Field, Ultramicroscopy 67 (1997), pp. 1-9.
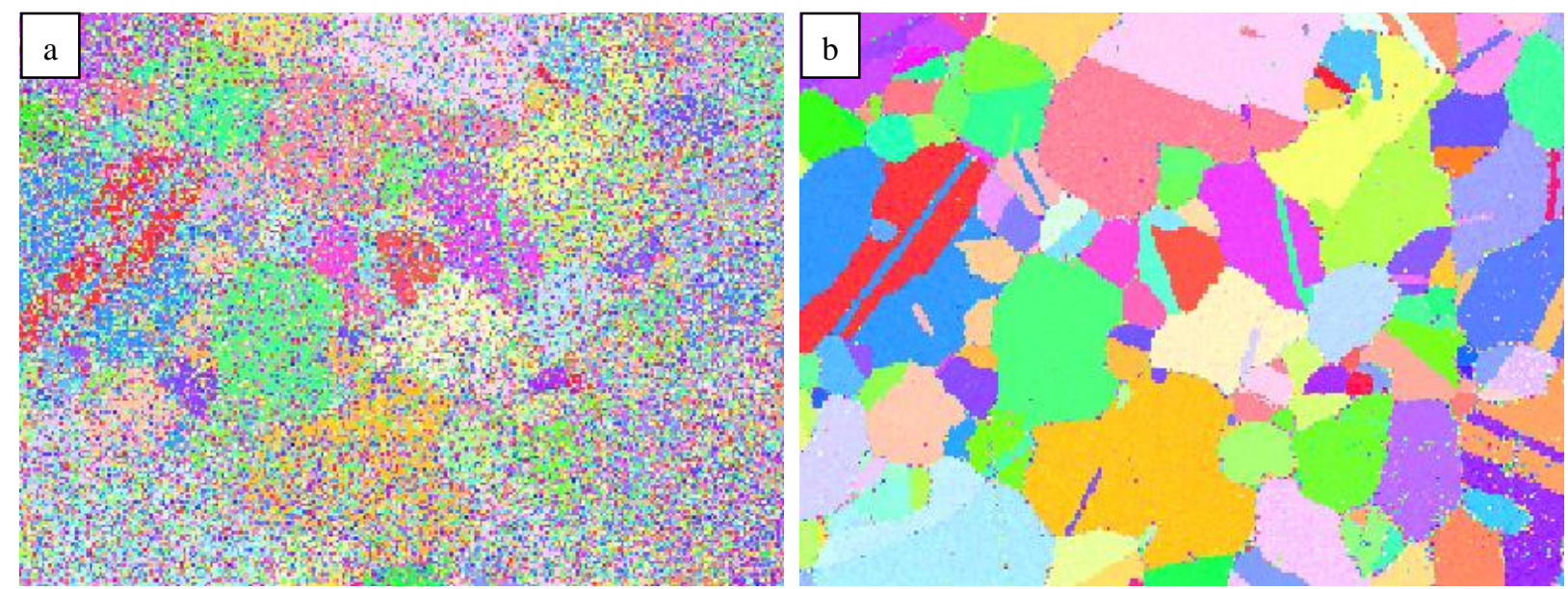

Figure 1. a) Orientation map reconstructed from EBSD patterns obtained on Nickel with 20 iterations of applied Poisson noise. b) Map reconstructed after applying the neighbor pattern averaging re-indexing algorithm.

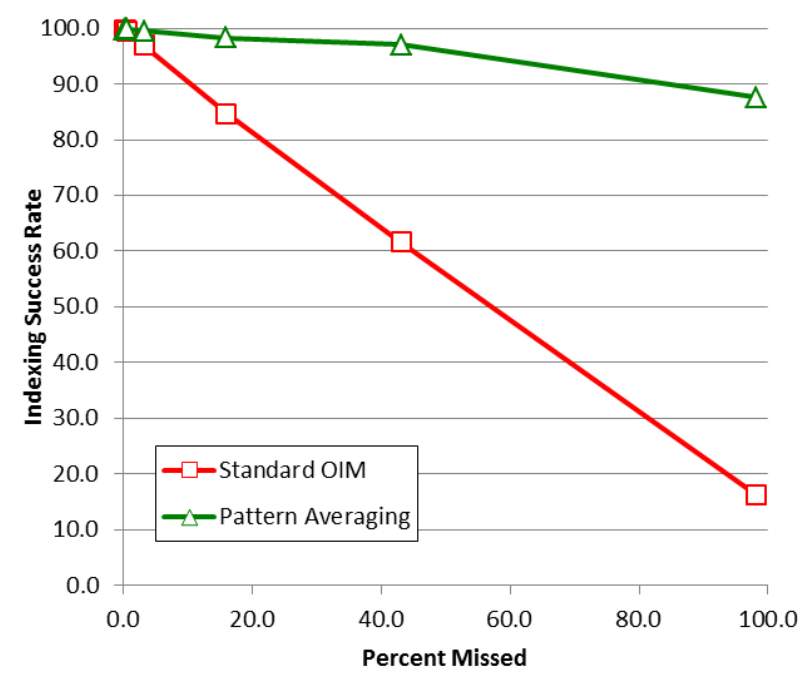

Figure 2. Indexing success rates for increasingly noisy camera conditions using the standard indexing routines and neighbor pattern averaging re-indexing. 